\title{
Alfabetización informacional en Bibliotecología y Ciencias de la Información Un análisis bibliométrico en el ámbito latinoamericano (2001-2020)
}

\section{Jesús Alberto Rivas Villena}

\begin{abstract}
Universidad Nacional Mayor de San Marcos. Escuela de Bibliotecología y Ciencias de la Información, Perú | jesus.rivas@unmsm.edu.pe / https://orcid.org/oooo-0002-6243-7721
\end{abstract}

\author{
Alejandro Uribe-Tirado \\ alejandro.uribe2@udea.edu.co / https://orcid.org/oooo-00o2-0381-1269

\section{Evony Katherine López-Mesa} \\ Universidad La Gran Colombia. Biblioteca, sede Bogotá, Colombia | \\ evony.lopez@ugc.edu.co / https://orcid.org/oooo-0oo1-9148-4001
}

Universidad de Antioquia. Escuela Interamericana de Bibliotecología, Medellín, Colombia |

\author{
Cesar H. Limaymanta \\ Universidad Nacional Mayor de San Marcos. Escuela de Bibliotecología y Ciencias de la Información / \\ Universidad Peruana de Ciencias Aplicadas. Área de Ciencias, Perú I \\ climaymantaa@unmsm.edu.pe | pcmaclim@upc.edu.pe | https://orcid.org/oooo-ooo2-8797-4275
}

\begin{abstract}
Resumen
Este artículo analiza la producción científica sobre ALFIN (2001-2020) en las áreas de Bibliotecología y Ciencias de la Información. A partir de Latindex, se identificaron todas las revistas de ByCI. Luego se verificó, si dichas revistas, estaban contenidas en las siguientes bases de datos: Web of Science (Core Collection y Scielo Citation Index), Scopus, Lens y Dimensions. Para la recuperación de registros sobre ALFIN y realizar el análisis bibliométrico, se eligió la base de datos Lens, por tener la mayor cobertura de revistas de ByCI en Latindex. Se evaluaron la tendencia y el crecimiento de la producción científica según autores y año de publicación; se analizó la productividad de los autores mediante la Ley de Lotka y la dispersión de la literatura según la Ley de Bradford. Se determinó el grado, índice y coeficiente de colaboración y se identificaron las redes de colaboración según autores. Los resultados muestran que la producción científica sobre ALFIN en Latinoamérica, alcanzó un pico entre los años 2017 y 2018, presentando un decrecimiento a partir del año 2019. Asimismo, se observó que la producción, colaboración entre autores y la cantidad de revistas es predominantemente brasileña.
\end{abstract}

Palabras clave

Bibliometría

Bibliotecología y Ciencia de la Información Alfabetización informacional Competencias informacionales América Latina Revistas científicas 
Information Literacy in Library and Information Science. A bibliometric analysis in the Latin American context (2001-2020)

\section{Abstract}

Keywords

Bibliometrics Library and Information Science Information Literacy Latin America Scientific journals Informational competences

This article analyzes the scientific production on ALFIN (2001-2020) in the areas of Library and Information Science. All BIC journals were identified from Latindex. Then it was verified whether these journals were contained in the following databases: Web of Science (Core Collection and Scielo Citation Index), Scopus, Lens and Dimensions. The Lens database was chosen for retrieving records on ALFIN and performing the bibliometric analysis, as it has the highest coverage of BIC journals in Latindex. The trend and growth of scientific production were evaluated according to authors and year of publication; the productivity of authors was analyzed using Lotka's Law and the dispersion of the literature according to Bradford's Law. The degree, index, and coefficient of collaboration were determined and collaboration networks were identified according to authors. The results show that scientific production on ALFIN in Latin America, reached a peak between 2017 and 2018, presenting a decrease from 2019 onwards. It was also observed that the production, collaboration among authors, and the quantity of journals is predominantly Brazilian.

Artículo recibido: 12-08-2021. Aceptado: 22-11-2021

\section{Introducción}

La Alfabetización Informacional -ALFIN- o las competencias informacionales-COINFO- (Sample, 2020; Landøy, Popa y Repanovici, 2020), como también se la conoce en América Latina, (Machin-Mastromatteo y Lau, 2015; Belluzzo, 2018), es una temática que durante las últimas décadas ha tenido abundante producción científica (artículos, libros, capítulos, etc.). Esto se ha identificado en distintos estudios métricos a nivel mundial (Park y Kim, 2011; Pinto, Escalona-Fernández y Pulgarín, 2013; Majid et al, 2015; Kolle, 2017; Stopar y Bartol, 2019; Onyancha, 2020; Li, Chen y Wang, 2021), lo cual evidencia su vigencia y uso.

1. https://en.unesco.org/ themes/media-and-information-literacy/resources

2. https://www.ifla.org/ information-literacy
La ALFIN es reconocida como una formación clave. En ese sentido, tanto la UNESCO ${ }^{1}$ y la IFLA ${ }^{2}$, consideran que, en educación, es totalmente necesario el uso de las nuevas tecnologías que permitan a los usuarios de información, conseguir las destrezas y aptitudes necesarias para comunicarse, interpretar y producir mensajes (Cisneros Rodríguez, García Dúctor y Lozano Jurado, 1999; Rader, 2002; Area Moreira y Guarro, 2012), utilizando distintos tipos de lenguajes y medios, que van desarrollándose en función al avance de las tecnologías (Area Moreira, 2008; García-Ávila, 2017).

El proceso de formación en ALFIN se enmarca, por tanto, en: definición del tema o necesidad, localización, evaluación, organización, comunicación y uso de información (Flores Noriega, 2011). Esto implica, que los usuarios, no solo deben estar alfabetizados en la gestión de la información; sino también en investigación, recursos, tecnología emergente, entre otros ámbitos. Estos aspectos contribuirán a una alfabetización integral, proactiva y no reactiva, de una multialfabetización. 
Como afirman Webber y Johnston (2002), se trata de una conducta informativa para obtener, a través de cualquier canal o medio, información adecuada, bajo una conciencia crítica sobre la importancia de su uso, tanto hábil como ético, en la sociedad. De forma general Ortoll, la define como "la habilidad de reconocer una necesidad de información y la capacidad de identificar, localizar, evaluar, organizar, comunicar y utilizar la información de forma efectiva, tanto para la resolución de problemas como para el aprendizaje a lo largo de la vida" (Ortoll, 2003: 3).

Es decir, la ALFIN busca desarrollar la autonomía personal y el pensamiento crítico, permitiendo capacitar adecuadamente a las personas para la toma de decisiones y emitir opiniones como ciudadanos, que permitan construir una sociedad justa e intercultural, donde se conviva con las innovaciones que vayan surgiendo (CILIP, 2018; Sales, 2020). Innovaciones donde las TIC, la Web 1.0, 2.0, 3.0, etc., se convierten en medios y fuentes de información y aprendizaje. En ese sentido, los profesionales e instituciones llamadas a fomentar esa formación, han sido y seguirán siendo los bibliotecólogos-bibliotecarios y las bibliotecas (Ponjuán, Pinto y Uribe-Tirado, 2015; Licea de Arenas, 2017).

En síntesis, la ALFIN ha propiciado un cambio en el rol educativo de las bibliotecas, de los bibliotecólogos-bibliotecarios (profesionales de la información) y la necesidad de tener una mayor formación al respecto. Esto propició un cambio en la forma de pensar y actuar de las instituciones y de los profesionales de la información, quienes han de reconocer e interiorizar el carácter interdisciplinario del proceso y la necesidad de apropiarse de conocimientos y procedimientos de otras disciplinas (Pinto y Uribe-Tirado, 2017). Es decir, para garantizar que un programa de ALFIN tenga éxito, se debe asegurar que los formadores tengan las competencias que la ALFIN requiere para el mundo de hoy, sea desde la educación universitaria (Uribe-Tirado, 2012; Amavizca Montaño, 2019), o no universitaria (Jiménez Rojo, 2020). Más aún, con los efectos de la pandemia de la covid-19, que tienen exigencias como: múltiples fuentes de información, redes sociales, fake news, posverdad, infodemia, etc. (Cortes Vera, 2019; Lewandowsky, 2020).

Por ello, no es de extrañar que desde la Bibliotecología y Ciencias de la Información-ByCI-, que es la disciplina en la que converge la formación universitaria y de actualización de estos profesionales, sea donde más se ha publicado sobre esta temática, en el contexto latinoamericano e internacional. Una evidencia de ello, es la información que presenta la base de datos Lens, a partir de su clasificación subject ${ }^{3}$, en la cual podemos confirmar esta situación en las primeras cinco disciplinas: Library and Information Sciences (3.647), Education (1.059), Information Systems (492), Health Informatics (139), Computer Science Applications (102).

Por eso, es clave conocer esa dinámica en CI, ya que los académicos y profesionales de la información que trabajan la ALFIN, constantemente comparten sus modelos, estándares, hallazgos de investigaciones, ideas de implementación y formatos. Aunque el término "Alfabetización Informacional" fue acuñado por primera vez por Paul Zurkowski en 1974, su alcance y cobertura se está perfeccionando continuamente, como se evidencia año a año, en el total de esta producción y en los resultados que presentan los distintos estudios métricos a nivel mundial, mencionados anteriormente.

Por tanto, basado en las necesidades relacionadas con el rápido desarrollo de las tecnologías y la sociedad de la información, los profesionales de la información están capacitando en competencias informacionales a todo tipo de usuarios para que sean más productivos y eficaces. Esto es un reto que ha aumentado con la pandemia y al cual se está respondiendo de diferentes maneras creativas y según cada contexto4. 


\section{Revisión de literatura}

En el contexto latinoamericano, se han presentado en los últimos años, solo algunos estudios sobre ALFIN. En primer lugar, a nivel de Hispanoamérica, Licea de Arenas (2009) analizó los indicadores bibliométricos y dio cuenta de las fortalezas y debilidades de la ALFIN. A nivel iberoamericano, Uribe-Tirado y Alhuay-Quispe (2017) analizaron desde determinadas fuentes el comportamiento de autores iberoamericanos sobre la ALFIN a través de indicadores bibliométricos y altmétricos. Mientras que, desde la mirada de un país específico, González-Valiente (2015) analizó el impacto mediante el conteo de citas recibidas de la literatura cubana sobre la ALFIN; igual tipo de análisis hace por su parte Belluzzo (2017) para el caso de Brasil.

No obstante, la existencia de estos estudios métricos, desde nuestros contextos, son pocos y por la dinámica misma de la producción de ALFIN, se hace necesario realizar nuevos trabajos que permitan:

»Actualizar los anteriores, ya que en tres o cuatro años cambia y aumenta la producción;

»Utilizar otras fuentes de información de producción y métricas, para lograr una mirada o comprensión diferente y/o más amplia; y especialmente,

" Lograr una mirada específica de la región y la disciplina, ya que dichos estudios no se han enfocado solo en la producción latinoamericana y en el área de Bibliotecología y Ciencias de la Información.

\section{Objetivos del estudio}

Considerando la importancia de la ALFIN desde la ByCI, para la formación de las actuales y futuras generaciones y dada la necesidad de conocer los avances teóricos y prácticos, el objetivo general del presente estudio es: analizar la producción científica sobre ALFIN a partir de revistas latinoamericanas indexadas en Latindex (2001-2020) en el área de ByCI.

Asimismo, los objetivos específicos son: (a) identificar la producción científica según año y autores, (b) analizar la productividad de los autores de la ALFIN mediante la Ley de Lotka y la dispersión de la literatura según la Ley de Bradford, (c) determinar el grado, índice y coeficiente de colaboración de los documentos, y (d) identificar las redes de colaboración según autores.

Alcanzar estos objetivos, permitirá que esta investigación sirva de base para analizar cómo la ALFIN ha cambiado en los últimos 20 años en nuestra región, qué tendencias se identifican y finalmente, seguir creciendo en la cualificación de esta formación necesaria desde años atrás.

Este panorama, es más relevante en un contexto de pandemia y pospandemia, donde estar bien informados y no caer en noticias falsas (fake news) es una necesidad. A su vez, debemos tener en cuenta que ahora la ciencia abierta es una realidad, que en el ámbito investigativo, llegó para quedarse (Uribe-Tirado et al, 2020) y esto implica, nuevos retos para la alfabetización informacional, que ya venían en camino:

A literacia da informação, em conclusão, assume se como uma ferramenta de aprendizagem essencial para o desenvolvimento da Ciência Aberta, potenciando e capacitando o entendimento crítico dos conteúdos, a par do desenvolvimento e do progresso da investigação (Lopes, Antunes y Sanches, 2018), 
pero que la realidad de la covid-19 y todas sus implicaciones en gestión de información e investigación, han resignificado mucho más.

\section{Metodología}

Este trabajo es un estudio cuantitativo con metodología bibliométrica. Latindex fue la fuente principal para la obtención de revistas latinoamericanas en el área de ByCI, ya que esta incluye revistas de investigación y divulgación científica que se editan en América Latina. Fueron cinco fases hasta la obtención de registros finales:

Fase 1: Acceso al directorio de Latindex bajo el área de Ciencias Sociales; subtemas: Bibliotecología y Ciencias de la Información. Se recuperaron 174 y 217 títulos únicos de revistas, respectivamente. Estos registros fueron exportados en formato ".xls" y se normalizaron, así se obtuvieron como producto final 197 revistas con afiliación latinoamericana que se encuentran indexadas en Latindex.

Fase 2: Validación y uso de las ecuaciones de búsqueda en cada base de datos (Tabla 1). Para la recuperación de los registros sobre ALFIN en revistas indexadas en Latindex, se formuló la estrategia de búsqueda en cuatro bases de datos: Scopus, Web of Science (Core Collection y Scielo Citation Index), Lens y Dimensions. Para identificar los términos más representativos de los conceptos relacionados a la ALFIN, se consultaron dichos términos en idioma inglés (information literacy) en el Tesauro de la UNESCO, tesauro de Biblioteconomía y Documentación y el tesauro de Ciencias de la Documentación. De estas, se extrajeron otros términos relacionados con la information literacy como: information competencies, information ability, informational competencies e information skills; que fueron empleadas en las ecuaciones de búsqueda. Para el caso de Dimensions y Lens se usaron términos en español y portugués debido a los criterios de indexación de las fuentes mencionadas.

Fase 3: Verificación e identificación de revistas en cada base de datos. Este proceso consistió en identificar las revistas de ByCI obtenidas de Latindex, en las cuatro bases de datos mencionadas. Para esto, se tomó el campo Source Title o Títulos de Revistas, de los archivos descargados y se normalizaron. Se identificaron un total de 9080 revistas.

Fase 4: Cruce de información de las 9080 revistas con las 197 que están indexadas en Latindex para obtener un resultado final de 40 revistas. Esta fase consistió en hacer una comparación minuciosa entre cada uno de los resultados obtenidos de las bases de datos y el listado de revistas de Latindex. En el caso de Scopus, se obtuvieron 11 revistas de ByCI que abordaron temas sobre la ALFIN, en Lens 40, Dimensions 28, en Web of Science Core Collection 12 y en WoS-Scielo 8 revistas. Ver tabla completa en Excel, hoja "tabla_anexo" (Zenodo: https://doi.org/10.5281/zenodo.5670305).

Fase 5: Recuperación de registros finales desde Lens. A partir de la fase anterior, la base de datos Lens es la que contiene la mayor cantidad de revistas (40), las cuales se muestran en la Tabla 4 y en la hoja "tabla_anexo" (Zenodo: https:// doi.org/10.5281/zenodo.5670305). Siguiendo nuestro criterio de mayor cantidad (cobertura), se eligió a Lens como la base de datos de la cual se toman los registros definitivos para hacer el análisis y evaluación de la data extraída. En Lens se aplicó la ecuación de búsqueda señalada en la Tabla 1. El período de tiempo fue en el rango 2001-2020. Las tipologías documentales consideradas fueron: Article, 
book chapter, conference proceedings. Los idiomas seleccionados fueron: español, inglés y portugués. Finalmente se filtraron los resultados a partir de las 40 revistas. Luego de aplicar los filtros y criterios mencionados anteriormente, se obtuvo un total de 367 registros que fueron exportados en formato RIS, CSV y BibTex. Una de las limitaciones que se identificó en Lens, fue en la falta de normalización de metadatos motivo por el cual implicó un trabajo manual para normalizar nombres de autores, revistas, instituciones, entre otras entidades.

Tabla 1. Ecuaciones de búsqueda y criterios de delimitación (2001-2020**

\begin{tabular}{|c|c|c|}
\hline Base de datos & Ecuaciones de búsqueda & Resultados obtenidos \\
\hline Scopus & 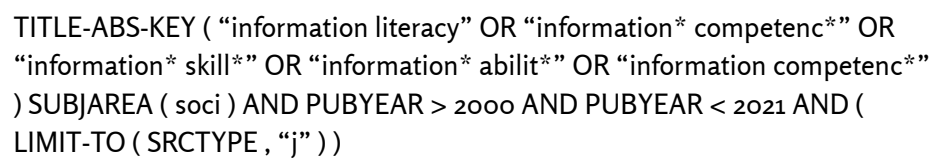 & $\begin{array}{l}5.730 \text { registros en } 11 \\
\text { revistas Latindex }\end{array}$ \\
\hline $\begin{array}{l}\text { Web of Science } \\
\text { (Core Collection } \\
\text { y Scielo Citation } \\
\text { Index) }\end{array}$ & $\begin{array}{l}\text { TS=(“information literacy" OR "information* competenc*" OR "information* } \\
\text { skill*” OR "information* abilit*") }\end{array}$ & $\begin{array}{l}4.096 \text { registros del Core } \\
\text { Collection ( } 12 \text { revistas } \\
\text { latindex) y } 235 \text { del Scielo } \\
\text { Citation Index (Ocho } \\
\text { revistas Latindex) }\end{array}$ \\
\hline Dimensions & $\begin{array}{l}\text { Title and Abstract: "information literacy" OR “information* competenc" } \\
\text { OR “information* skills" OR “information" abilit”" OR "alfabetización } \\
\text { informacional” OR "competencia" informacional"” OR “literacia da } \\
\text { informacao" OR “competencia da informacao" }\end{array}$ & $\begin{array}{l}9.511 \text { registros en } 28 \\
\text { revistas Latindex }\end{array}$ \\
\hline Lens & 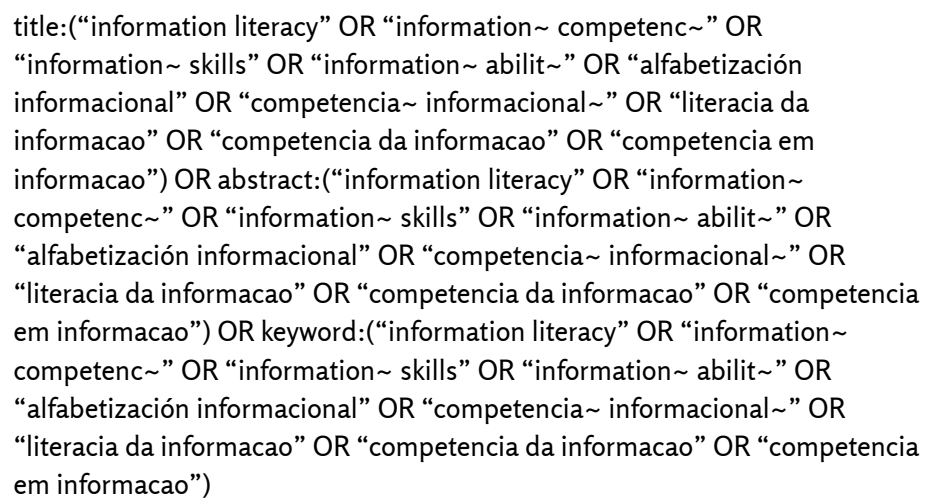 & $\begin{array}{l}34.7767 \text { registros en } 40 \\
\text { revistas Latindex }\end{array}$ \\
\hline
\end{tabular}

\footnotetext{
* La fecha de corte para la exportación de datos desde Lens, fue en junio de 2021.
}

Para obtener los indicadores bibliométricos se usaron los programas Publish or Perish 7, Excel 2019 y VOSviewer 1.6.17. En la tabla 2 se presentan los indicadores analizados con su respectiva descripción. 


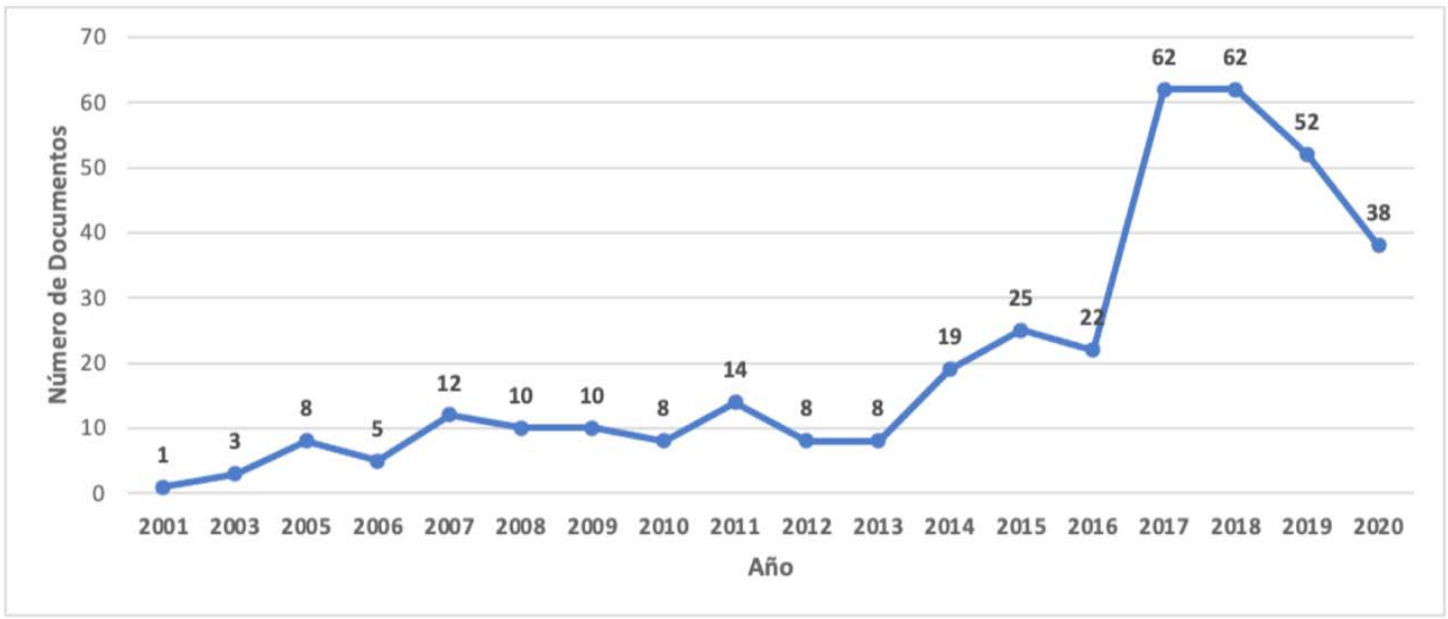

Nota: En función a la revisión realizada, la disminución de la producción en los últimos dos años se explica por dos razones: revistas que se atrasan en la publicación de artículos y la falta de actualización de los artículos indexados en Lens.

Tabla 2. Descripción de los indicadores bibliométricos y herramienta de análisis

\section{Dimensiones analizadas Indicadores/variables: Descripción}

Productividad científica Número de documentos: Calculado por año y por tipología

Ley de Lotka de la productividad de los autores

Dispersión de la literatura según la Ley de Bradford

Colaboración científica Índice de colaboración

Grado de colaboración

Coeficiente de colaboración

Análisis estructural

Red bibliométrica

Estructura social

Red de coautoría entre autores
Figura 1. Evolución de la producción científica de artículos (2001-2020)

\section{Resultados}

\section{Producción científica según año y autores más productivos (2001-2020)}

A continuación se presentan los resultados más relevantes obtenidos de la data extraída de la base de datos Lens. En primer lugar, se presenta la evolución de la producción científica desde 2001 hasta 2020. En la Figura 1, se observa una tendencia creciente en la producción científica de los artículos, el pico se encuentra en los años 2017 y 2018 con 62 documentos publicados. Se hicieron revisiones en otras bases de datos sobre este aspecto, sin embargo; no se evidenciaron tendencias significativas que permitan afirmar con certeza la causa de dicho incremento en estos años. Probablemente, se deba a la inclusión de documentos, materia de estudio, que fueron indexadas por Lens. Es importante resaltar que a partir de 2018, la producción científica decreció, ya que para el año 2019 dicha producción decayó en 16\%. A este respecto, se realizó 
la revisión correspondiente y se concluye que, probablemente, se deba al tiempo de desfase que presenta Lens en la indexación de algunos artículos en este periodo.

\section{Autores más productivos}

Para visualizar el reconocimiento de los autores más productivos (Tabla 3) de la comunidad examinada, se analizó el número total de artículos que cada autor publicó (ND). Además, para cada autor se consideró otros campos como: institución y país.

\section{Productividad de los autores según la Ley de Lotka}

En la figura 2 se presenta la distribución de la productividad de los 473 autores provenientes de los documentos analizados. En la parte izquierda se presenta la distribución de autores según número de documentos publicados. Así, se evidencia que existen 354 autores con un solo documento publicado, 65 autores con dos publicaciones cada uno y así sucesivamente. Asimismo, en la parte inferior de la tabla se observa que existe un autor con 20 publicaciones, otro autor con 17 documentos y otro autor con 15 documentos. Se cumple que, un grupo reducido de autores publican un mayor volumen de documentos sobre ALFIN en revistas Latindex del ámbito latinoamericano y que están en Lens (Tabla 3), en tanto que la mayoría de ellos, llamados autores transitorios, registra poca productividad. En la parte derecha se observa el modelo inverso de Lotka con pendiente -2.023 e índice de bondad de ajuste (coeficiente de determinación) de $\mathrm{R} 2=92.1 \%$, el cual indica que el modelo se ajusta a los datos analizados.

Tabla 3. Top 15 autores más productivos según número de documentos, institución y país

\begin{tabular}{|c|l|c|l|l|}
\hline $\mathbf{N}^{\circ}$ & Autores & ND & Institución & País \\
\hline 1 & Elizete Vieira Vitorino & 20 & Universidade Federal de Santa Catarina & Brasil \\
\hline 2 & Regina Célia Baptista Belluzzo & 17 & Universidad Estadual Paulista & Brasil \\
\hline 3 & Selma Leticia Capinzaiki Ottonicar & 15 & Universidade Estadual Paulista & Brasil \\
\hline 4 & Helen de Castro Silva Casarin & 10 & Universidade Estadual Paulista & Brasil \\
\hline 5 & Alejandro Uribe-Tirado & 10 & Universidad de Antioquia & Colombia \\
\hline 6 & Gustavo Henrique de Araújo Freire & 9 & Universidade Federal do Ceará & Brasil \\
\hline 7 & Jussara Borges & 8 & Universidade Federal da Bahia & Brasil \\
\hline 8 & Gabriela Belmont de Farias & 8 & Universidade Federal do Ceará & Brasil \\
\hline 9 & Maria Giovanna Guedes Farias & 8 & Universidade Estadual Paulista & Brasil \\
\hline 10 & Rafaela Carolina da Silva & 7 & Universidade Estadual Paulista & Brasil \\
\hline 11 & Grizly Meneses-Placeres & 7 & Universidad Central “Marta Abreu” de Las Villas & Cuba \\
\hline 12 & Elmira Luzia Melo Soares Simeão & 7 & Universidad de Brasilia & Brasil \\
\hline 13 & Marta Lígia Pomim Valentim & 7 & Universidade do Rio de Janeiro & Brasil \\
\hline 14 & Isa Maria Freire & 6 & Universidade Federal da Paraíba Nenhum & Brasil \\
\hline 15 & Carlos Luis González Valiente & 6 & European Alliance for Innovation & Cuba \\
\hline
\end{tabular}




\begin{tabular}{|c|c|}
\hline ND & NA \\
\hline 1 & 354 \\
\hline 2 & 65 \\
\hline 3 & 16 \\
\hline 4 & 17 \\
\hline 5 & 6 \\
\hline 6 & 2 \\
\hline 7 & 4 \\
\hline 8 & 3 \\
\hline 9 & 1 \\
\hline 10 & 2 \\
\hline 15 & 1 \\
\hline 17 & 1 \\
\hline 20 & 1 \\
\hline Total & 473 \\
\hline
\end{tabular}

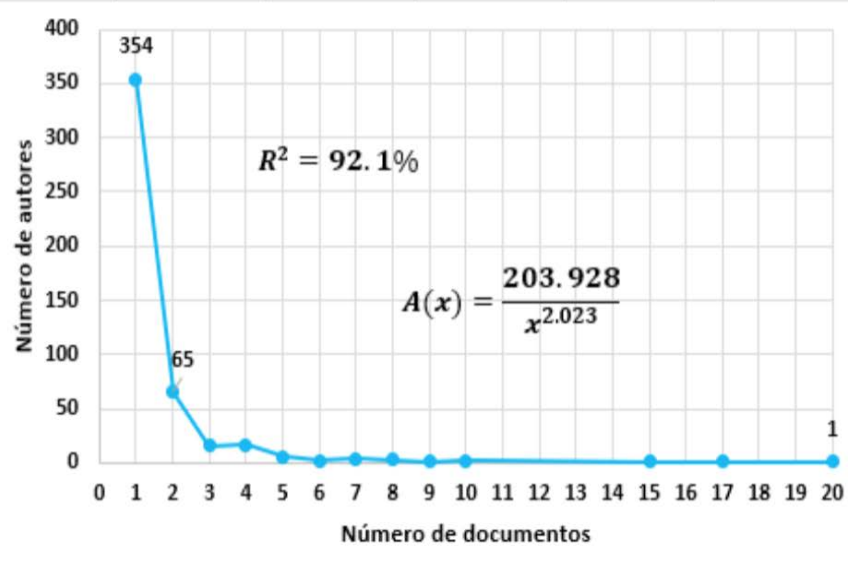

Nota: ND: Número de documentos, NA: Número de autores

\section{Dispersión de la revistas según la Ley de Bradford}

En cuanto a la distribución de revistas latinoamericanas que publicaron sobre la ALFIN, la Tabla 4 presenta las revistas ordenadas según el número de documentos publicados. Siguiendo el enunciado de la Ley de Bradford (Desai, Veras y Gosain, 2018) existen dos revistas en el núcleo que reúnen aproximadamente la tercera parte de todos los documentos $(127 ; 34.6 \%)$, siendo Pesquisa Brasileira em Ciencia da Informacao e Biblioteconomia la revista más productiva con 87 documentos. En la zona 2 se encuentran ocho revistas que acumulan 133 documentos (36.2\%) y en la zona 3 existen 30 revistas menos productivas que agrupan a 107 documentos (29.2\%). Del total de 40 revistas se observa la predominancia de revistas brasileñas en la producción científica sobre ALFIN (26; 65\%). Por ello, gran parte del desarrollo de esta categoría de conocimiento se debe al aporte de las revistas brasileñas. Le siguen Cuba con tres revistas, Argentina, Costa Rica y México con dos revistas cada una.

\section{Grado, índice y coeficiente de colaboración}

La Figura 3, muestra los indicadores de colaboración de los documentos producidos en el periodo de la investigación. Respecto al grado de colaboración, es relevante la concentración de documentos producidos en colaboración, es decir por dos o más autores (66\%); frente al $34 \%$ de los documentos fueron producidos por autores únicos. El índice de colaboración refleja que, en promedio, la cantidad de autores por artículo es de 1,9 autores. En el caso del coeficiente de colaboración, se observa que, en general, se ha incrementado sostenidamente en los últimos años, siendo el 2020 el año que presenta el mayor nivel de dicho coeficiente con $54 \%$.

Figura 2. Productividad de autores según número de documentos publicados con la ecuación del modelo inverso de Lotka 
Figura 3. Índice, grado y coeficiente de colaboración de documentos

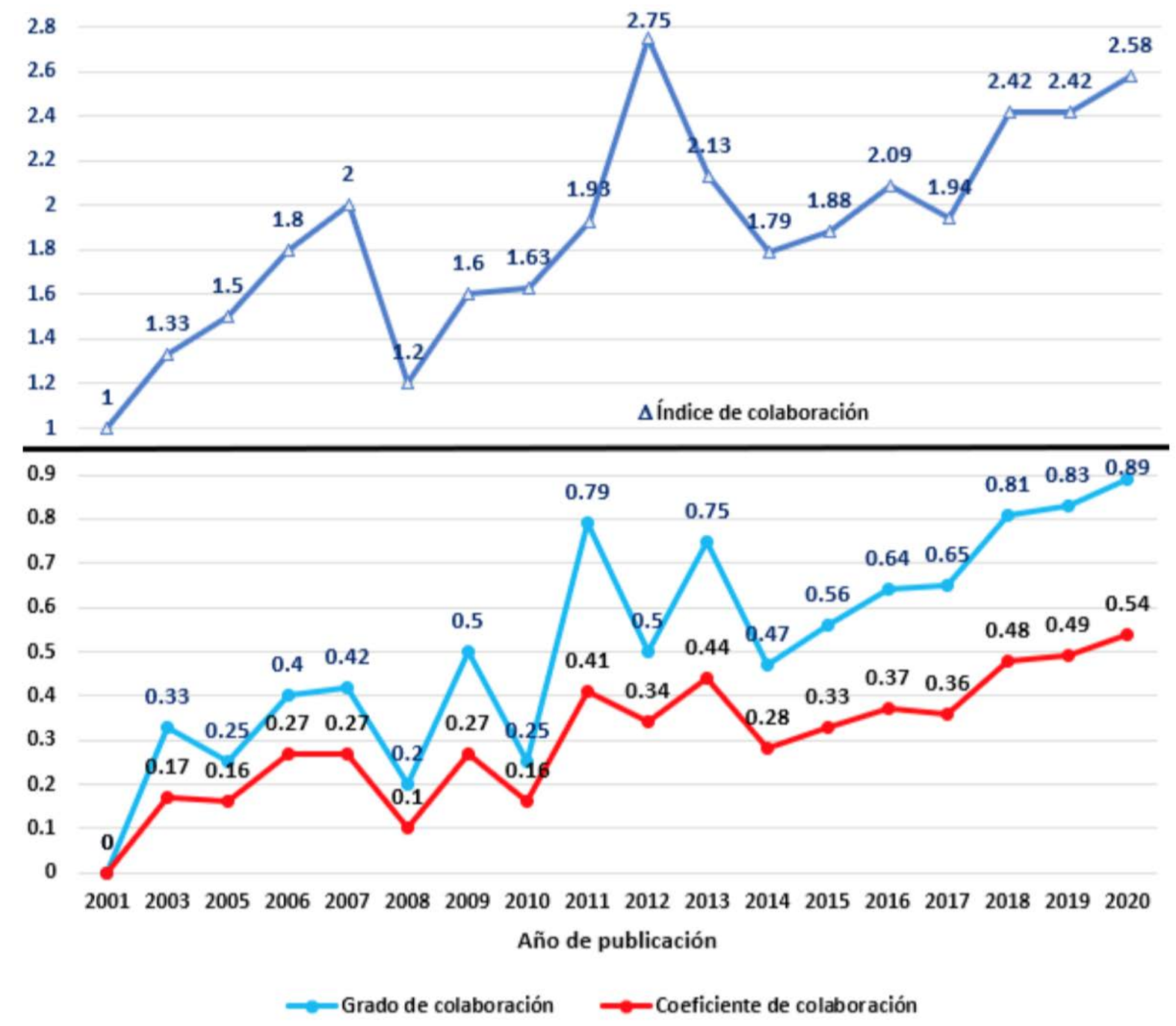


Tabla 4. Dispersión de las Revistas Latindex sobre ALFIN en Latinoamérica según la Ley de Bradford

\begin{tabular}{|c|c|c|c|c|}
\hline $\mathbf{N}^{\circ}$ & Revista & País & ND & $\begin{array}{l}\text { Zona/NR/ND } \\
\text { Ley de Bradford }\end{array}$ \\
\hline 1 & Pesquisa Brasileira em Ciência da Informação e Biblioteconomia & Brasil & 87 & \multirow{2}{*}{$\begin{array}{l}\text { Zona } 1 \text { o núcleo } \\
\text { Dos revistas } \\
127 \text { documentos }\end{array}$} \\
\hline 2 & ACIMED-Revista Cubana de Información en Ciencias de la Salud & Cuba & 40 & \\
\hline 3 & Informação \& sociedade: Estudos & Brasil & 32 & \multirow{8}{*}{$\begin{array}{l}\text { Zona } 2 \\
\text { Ocho revistas } \\
133 \text { documentos }\end{array}$} \\
\hline 4 & Revista Ibero-Americana de Ciência da Informação & Brasil & 21 & \\
\hline 5 & Revista Interamericana de Bibliotecología & Colombia & 18 & \\
\hline 6 & Encontros Bibli: revista eletrônica de biblioteconomia e ciência da informação & Brasil & 13 & \\
\hline 7 & Perspectivas em Ciência da Informação & Brasil & 13 & \\
\hline 8 & Bibliotecas: Anales de Investigación & Cuba & 12 & \\
\hline 9 & Em Questão & Brasil & 12 & \\
\hline 10 & Investigación Bibliotecológica: archivonomía, bibliotecología e información & México & 12 & \\
\hline 11 & Informação \& Informação & Brasil & 11 & \multirow{30}{*}{$\begin{array}{l}\text { Zona } 3 \\
30 \text { revistas } \\
107 \text { documentos }\end{array}$} \\
\hline 12 & Ciencias de la Información & Cuba & 9 & \\
\hline 13 & Información, cultura y sociedad & Argentina & 9 & \\
\hline 14 & Revista Brasileira de Educação em Ciência da Informação & Brasil & 9 & \\
\hline 15 & Biblios & Perú & 6 & \\
\hline 16 & e-Ciencias de la información & Costa Rica & 6 & \\
\hline 17 & InCID: Revista de Ciência da Informação e Documentação & Brasil & 6 & \\
\hline 18 & Brazilian Journal of Information Science: Research Trends & Brasil & 5 & \\
\hline 19 & Biblionline & Brasil & 4 & \\
\hline 20 & Ponto de Acesso & Brasil & 4 & \\
\hline 21 & RDBCI: Revista Digital de Biblioteconomia e Ciência da Informação & Brasil & 4 & \\
\hline 22 & Transinformação & Brasil & 4 & \\
\hline 23 & Informação@Profissões & Brasil & 3 & \\
\hline 24 & ReHuSo: Revista de Ciencias Humanísticas y Sociales & Ecuador & 3 & \\
\hline 25 & Serie Bibliotecología y Gestión de Información & Chile & 3 & \\
\hline 26 & Archeion Online & Brasil & 2 & \\
\hline 27 & AtoZ: novas práticas em informação e conhecimento & Brasil & 2 & \\
\hline 28 & Biblioteca Universitaria & México & 2 & \\
\hline 29 & BIBLOS: Revista da Faculdade de Letras da Universidade de Coimbra & Brasil & 2 & \\
\hline 30 & Logeion: Filosofia da Informação & Brasil & 2 & \\
\hline 31 & Palabra Clave (La Plata) & Argentina & 2 & \\
\hline 32 & Ãgora & Brasil & 1 & \\
\hline 33 & Acervo & Venezuela & 1 & \\
\hline 34 & Bibliotecas: Revista de la Escuela de Bibliotecología, Documentación e Información & Costa Rica & 1 & \\
\hline 35 & BIBLOS - Revista do Instituto de Ciências Humanas e da Informação (ICHI) & Brasil & 1 & \\
\hline 36 & Cadernos de Educação Tecnologia e Sociedade & Brasil & 1 & \\
\hline 37 & Ciência da Informação & Brasil & 1 & \\
\hline 38 & Comunicação \& Informação & Brasil & 1 & \\
\hline 39 & InfoDesign - Revista Brasileira de Design da Informação & Brasil & 1 & \\
\hline 40 & South American Development Society Journal & Brasil & 1 & \\
\hline
\end{tabular}

Nota: NR: Número de revistas; ND: Número de documentos 
Figura 4. Visualización de la red de coautoría entre autores

zattar, marianna

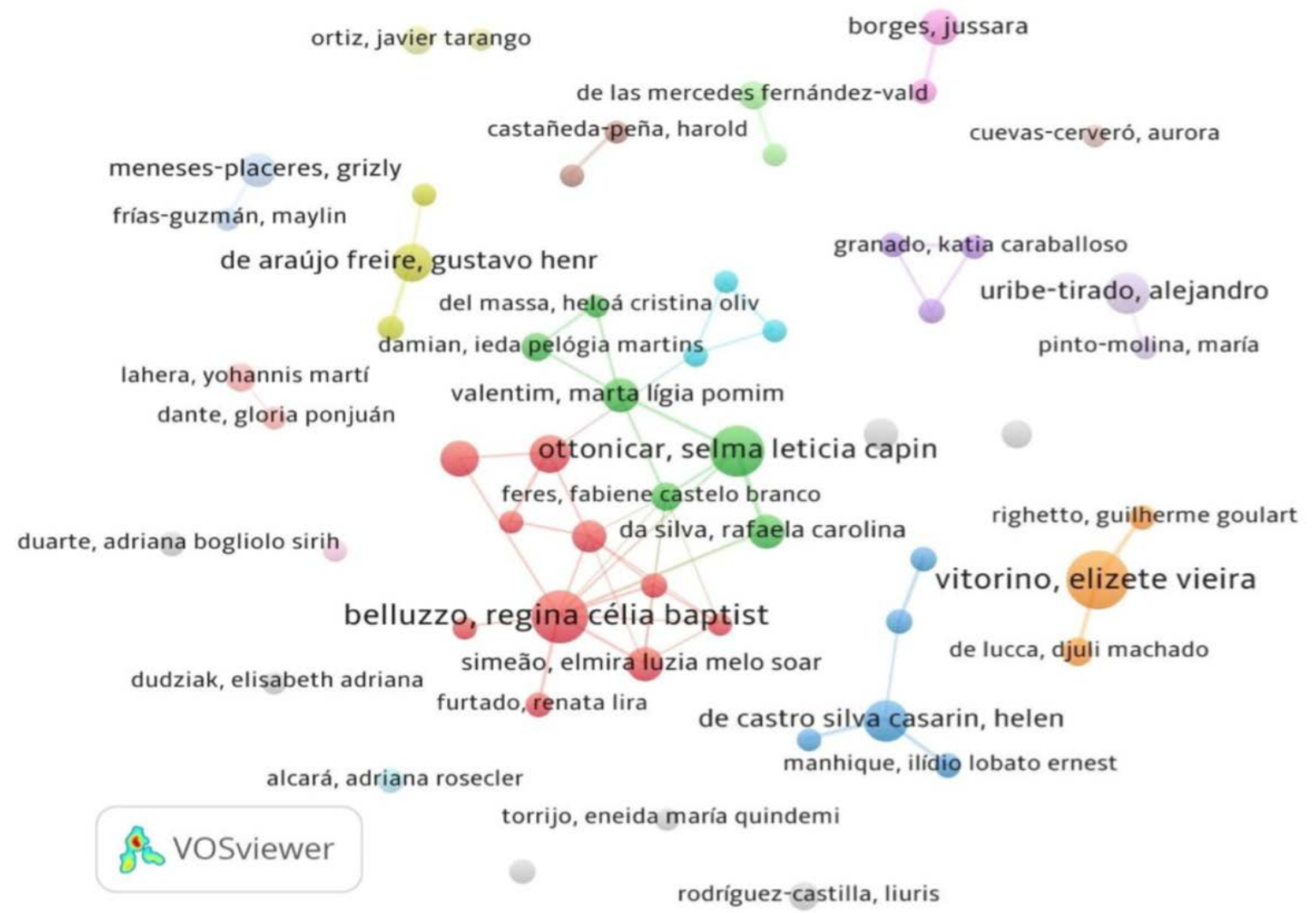

Nota: se tuvo en cuenta autores mínimo dos documentos $(n=3)$. De los 460 autores, 59 cumplen con el umbral. Método de conteo: fractional counting; atracción: 2; repulsión: -3; resolución de agrupamiento: 0.5.

\section{Red de coautoría}

La figura 4 muestra 26 clústeres (reflejados cada uno con un color distinto) que representan las diferentes colaboraciones de los autores que han contribuido sobre la ALFIN. Cabe resaltar que las autoras Regina Célia Baptista Belluzzo y Selma Leticia Capinzaiki Ottonicar, quienes se encuentran dentro de las autoras más productivas, además presentan colaboración entre las diferentes agrupaciones, convirtiéndose en las autoras con mayor influencia en la especialidad temática. También se encuentra la autora Elizete Vieira Vitorino con una gran concentración de productividad representativa en la ALFIN, aunque ella solo presenta colaboración con los autores Guilherme Goulart Righetto y Djuli Machado de Lucca (autores con al menos tres documentos dentro del mapa).

La mayor parte de los documentos publicados en colaboración se concentran en instituciones de un mismo país (Brasil). Sin embargo, también se evidencia colaboración y 
producción de otros países en ALFIN como es el caso de Colombia representado por el autor Alejandro Uribe-Tirado, quien hace colaboración con la autora española María Pinto Molina y para Cuba, en el caso de las autoras Maylín Frías-Guzmán y Grizly Placeres-Meneses. Además, se evidencia la presencia de algunos autores mexicanos como Javier Tarango, entre otros.

\section{Discusión y conclusiones}

Los datos anteriores dan cuenta de la realidad más actual de la producción científica sobre la ALFIN desde revistas latinoamericanas de ByCI. Producción que, durante los últimos años, venía mostrando un crecimiento sostenido, sin embargo, ha tenido variaciones que tienden a la baja y se deberían analizar en próximos años (pospandemia). Esto, con el fin de identificar si habían cambios en la publicación por las dinámicas investigativas y prácticas de esta temática (años: 2019-2020; Figura 1), independientemente de la pandemia o por ella misma (a partir de marzo de 2020, para nuestros países) y sus efectos positivos o negativos en dicha dinámica de investigaciónaplicación-producción. Tener una o varias razones para ese decrecimiento, habrá que indagarlas más adelante, ya que podría deberse a los tiempos de indexación en las bases de datos consultadas y las revistas consideradas, o más recientemente, a los efectos del inicio y desarrollo de la pandemia (marzo de 2020), que afectó las dinámicas investigativas, pero esto sería motivo de otro estudio, necesario hacer más adelante.

Con relación a los datos presentados, específicamente a la producción científica, se identificó un total de 367 documentos desde 2001 hasta 2020, siendo los últimos cuatro (especialmente antes de la pandemia), los que muestran los niveles más altos. Esto demuestra la trascendencia que seguían tomando las investigaciones sobre la ALFIN a finales de la última década. Por otro lado, se comprobó que los autores más productivos (Tabla 3), están compuestos en su mayoría, por investigadores brasileños (80\%), esto patenta y posiciona a Brasil como el país líder en producción científica en ALFIN en el área de Bibliotecología y Ciencias de la Información en Latinoamérica.

El análisis de la distribución de los autores según su productividad, revela el cumplimiento del modelo de Lotka, también conocido como el efecto mateo de la producción científica. Es decir, hay un número pequeño de autores que concentran la mayor parte de la producción científica. Mientras que hay una gran cantidad de autores transitorios con pocas publicaciones. A partir de allí, se espera que los autores más productivos sigan influenciando en el desarrollo de este dominio de ALFIN en revistas latinoamericanas de ByCI, pues la formación en competencias informacionales, como se ha indicado, es muy necesaria en estos tiempos de explosión de la información (social y académica, falsa y real) en distintas plataformas digitales. En cuanto a las revistas, gracias a los enunciados de la ley de Bradford, se logró identificar a las revistas más productivas que se encuentran en el núcleo y en las zonas periféricas. El protagonismo se lo llevan las revistas brasileñas con más del $60 \%$ de presencia en el conjunto de ellas. Gracias a ambos resultados se demuestra la vigencia de estas leyes formuladas en el siglo pasado por Lotka (1926) y Bradford (1934).

Respecto al Grado de Colaboración (GC), en promedio, el 66\% de documentos fueron publicados en colaboración (dos o más autores), evidenciando que la producción científica en cuestión, ha sido realizada colaborativamente sobre aquellos documentos elaborados individualmente, sobre todo en los últimos años. Esto refleja el protagonismo que ha tomado la colaboración entre investigadores en materia de la ALFIN. Por otra parte, el Índice de Colaboración (IC) muestra un promedio de 1.9 autores por artículo, mostrando una tendencia ascendente hacia el año 2020. Además, en el año 2001 el IC fue 1, mientras que, en el 2020 fue 2.58, lo cual demuestra que la cantidad 
de investigadores involucrados en un documento tiende a ser mayor. En relación al Coeficiente de Colaboración (CC), también ha mostrado un crecimiento en los últimos años (0.48, 0.49 y 0.54 en 2018, 2019 y 2020, respectivamente), lo cual refleja que los documentos elaborados individualmente, están disminuyendo. De acuerdo a la red de coautoría entre autores, se deduce que el tema estudiado presenta una mayor colaboración nacional, debido a las instituciones a las que pertenecen, las afiliaciones de los autores (Brasil) y el grado de contribuciones entre ellos. Sin embargo, también se resalta la colaboración de países iberoamericanos como es el caso de México, Colombia, España y Cuba, aspecto importante a tener en cuenta al abordar estudios de caso sobre la ALFIN.

Los textos y reflexiones más recientes, indican que esta formación estará marcada por dos grandes tendencias: por un lado, a) el reto de seleccionar información de calidad para el desarrollo académico-investigativo (difusión), ante el crecimiento exponencial de la producción científica y cada vez más colaborativa. Pero especialmente para la información ciudadana (divulgación-apropiación social del conocimiento), evitando así, las noticias falsas en cualquier tema y b) la formación será cada vez más mediadas por las TIC's, ya que la ALFIN se viene apoyando cada vez más en ellas (mixta, totalmente virtual, e-mobile, tutoriales en línea, etc.), pero es evidente que, estos últimos años, su dinámica es y será cada vez más creciente. Las TIC's, llegaron para quedarse y no solo para la aplicarse en la mediación-formación, como era antes de la pandemia, sino para ser la principal manera de formación en competencias informacionales, es decir, la E-ALFIN llegó para quedarse en todos los contextos.

Respecto a los resultados obtenidos a nivel mundial, se encuentran estudios bibliométricos los cuales abarcan periodos a partir de 1991 hasta 2019, (Park y Kim, 2011; Pinto, Escalona-Fernández y Pulgarín, 2013; Majid et al, 2015; Kolle, 2017; Stopar y Bartol, 2019) en el que se emplean las bases de datos Web of Science y Scopus, donde se evidencia países más productivos como Estados Unidos, Reino Unido y España (en este último María Pinto Molina es la autora más visible). Por otra parte, Onyancha (2020) Li, Chen y Wang (2021) en sus trabajos a partir de estudios métricos reflejan cómo el término "information literacy" se va acuñando con el paso de los años y la productividad en esta temática aumenta.

A nivel de Iberoamérica, se encuentra el estudio de Licea de Arenas (2009), en el cual se identifica el análisis de algunos indicadores de producción y concluye que el país más productivo en ALFIN, es Brasil. Por otra parte, una de las autoras más destacadas en este estudio, es la brasileña Regina Célia Baptista Belluzzo. Sin embargo, esta investigación no menciona el periodo de tiempo en el que se analiza la información y solo menciona los artículos que han sido indizados en las fuentes de datos Web of Knowledge y Scopus.

Asimismo, Uribe-Tirado y Alhuay-Quispe (2017), realizaron una investigación bajo un análisis con indicadores bibliométricos tradicionales, en el que se evidencia que el país más productivo es España (esto se debe a que el estudio se realiza a nivel Iberoamérica). Aunque Brasil está en segundo lugar, reafirmando lo que se menciona en nuestra investigación; este análisis se realiza en diversas fuentes de datos como Google Scholar, Scopus, Web of Science y Microsoft Academic. Mientras que en el documento de González-Valiente (2015) se realiza un análisis bibliométrico con indicadores de impacto, el cual se basa en la producción cubana en ALFIN de los años 2000-2013, donde se resalta la temática estudiada en el área de la salud. 
Dicho lo anterior, el presente estudio refleja un análisis más descriptivo y se incluyen no solo indicadores de producción, sino de colaboración desde la base de datos Lens, que es de acceso abierto, lo cual permite tener otra perspectiva en el abordaje de investigaciones bibliométricas. En la cual tienen predominancia otras fuentes, como Web of Science y Scopus, los cuales son tradicionalmente empleados para realizar este tipo de estudios.

\section{Agradecimientos}

Al grupo de investigación CIGETMEN de la Universidad Nacional Mayor de San Marcos y a la Dirección de Investigación de la Universidad Peruana de Ciencias Aplicadas. 


\section{Q Referencias bibliográficas}

"Amavizca Montaño, Sofía, coord. 2019. Alfabetización Informacional para la gestión del conocimiento en la Universidad. México: Universidad Estatal de Sonora. <https://gredos.usal.es/bitstream/handle/10366/142971/DBD_Alfindigital. pdf? sequence $=6 \&$ isAllowed $=y>$ [Consulta: 17 febrero 2021] .

" Area Moreira, Manuel. 2008. La innovación pedagógica con TIC y el desarrollo de las competencias informacionales y digitales. En Investigación en la escuela. No.64, 5-17. <https://revistascientificas.us.es/index.php/IE/article/view/7157> [Consulta: 17 febrero 2021].

»Area Moreira, Manuel y Amador Guarro. 2012. La alfabetización informacional y digital: fundamentos pedagógicos para la enseñanza y el aprendizaje competente. En Revista Española de Documentación Científica. No. 35, 46-74. <https:// doi.org/10.3989/redc.2012.mono.977>

» Belluzzo, Regina Celia Baptista. 2017. O estado da arte da competência em informação (Colnfo) no Brasil: das reflexões iniciais à apresentação e descrição de indicadores de análise. En Revista Brasileira de Biblioteconomia e Documentação. No. 13, 47-76. <https://rbbd.febab.org.br/rbbd/article/ view/648> [Consulta: 17 febrero 2021].

》Belluzzo, Regina Celia Baptista. 2018. Competência em informação no Brasil: cenários e espectros. São Paulo: ABECIN.

" CILIP definition of information literacy 2018. 2018. Chartered Institute of Library and Information Professionals Information Literacy Group. <https://infolit.org. uk/ILdefinitionCILIP2018.pdf> [Consulta: 17 febrero 2021].

"Cisneros Rodríguez, Inés, Catalina García Dúctor e Isabel María Lozano Jurado. 1999. ¿Sociedad de la información. Sociedad del conocimiento? La educación como mediadora. En EDUTEC 99. IV Congreso de Nuevas Tecnologías de la Información y de la Comunicación para la educación. Nuevas tecnologías en la

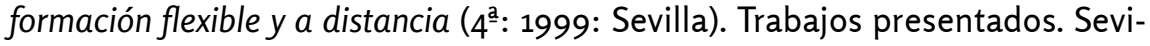
Ila, España: Universidad de Sevilla. Secretariado de Recursos Audiovisuales y Nuevas Tecnologías. <https://idus.us.es/handle/11441/62692> [Consulta: 17 febrero 2021].

»Cortes Vera, Jesús. 2019. La alfabetización informacional, bastión en tiempos de la posverdad. En Bibliotecas. Anales de Investigación. Vol. 15, no.3, 412420. <http://cathi.uacj.mx/handle/20.500.11961/10888> [Consulta: 17 febrero 2021].

»Desai, Nidhi, Laura Veras y Ankush Gosain. 2018. Using Bradford's law of scattering to identify the core journals of pediatric surgery. En Journal of Surgical Research. No. 229, 90-95. <https://doi.org/10.1016/j.jss.2018.03.062>

"Flores Noriega, Eva. 2011. Biblioteca y docencia: motivando el desarrollo de un programa ALFIN en el Consorcio de Universidades. En Alexandria: revista de Ciencias de la Información. Vol. 5, no. 8, 54-68. <http://revistas.pucp.edu.pe/ index.php/alexandria/article/download/216/210/> [Consulta: 17 febrero 2021].

»García-Ávila, Susana. 2017. Alfabetización digital. En Razón y Palabra. Vol. 21, no. 3, 66-81. <https://www.revistarazonypalabra.org/index.php/ryp/article/ view/1043> [Consulta: 17 febrero 2021]. 
» González-Valiente, Carlos Luis. 2015. Una aproximación al impacto de la investigación cubana sobre alfabetización informacional. En Revista Cubana de Información en Ciencias de la Salud. Vol. 26, no.1, 53-70. <http://scielo.sld.cu/scielo.php?script=sci_ar ttext\&pid=S2307-21132015000100006> [Consulta: 17 febrero 2021].

» Jiménez Rojo, Ángel. 2020. La competencia informacional y el pensamiento crítico en la enseñanza no universitaria: una revisión sistemática. En Revista Interuniversitaria de Investigación en Tecnología Educativa. No. 9, 1-18. <https:// doi.org/10.6018/riite.431381>

"Kolle, Shankar Reddy. 2017. Global research on information literacy: a bibliometric analysis from 2005 to 2014. En The Electronic Library. Vol. 35, no. 2, 283298. <https://doi.org/10.1108/EL-08-2015-0160>

"Landøy, Ane, Daniela Popa y Angela Repanovici. 2020. Basic concepts in information literacy. En Landøy, Ane, Daniela Popa y Angela Repanovici. Collaboration in designing a pedagogical approach in information literacy. Cham: Springer. p. 23-38.

"Lewandowsky, Stephan. 2020. The 'post-truth'world, misinformation, and information literacy: a perspective from cognitive science. En Goldstein, Stéphane, ed. Informed Societies: Why information literacy matters for citizenship, participation and democracy. Cambridge: Facet Publishing. p. 69-88.

"Li, Yating, Ye Chen y Qiyu Wang. 2021. Evolution and diffusion of information literacy topics. En Scientometrics. Vol. 126, no. 5, 4195-4224. <https://doi. org/10.1007/s11192-021-03925-y>

» Licea de Arenas, Judith. 2009. La Alfabetización Informacional en el entorno Hispanoamericano. En Anales de Documentación. Vol. 12, 93-106. <https://revistas.um.es/analesdoc/article/view/70261> [Consulta: 17 febrero 2021].

»Licea de Arenas, Judith. 2017. Para (re) pensar la alfabetización informacional en la universidad. En Biblioteca Universitaria. Vol. 20, no. 1, 23-33. <https://doi. org/10.22201/dgb.0187750xp.2017.1.173>

» Lopes, Carlos Alberto, Maria da Luz Antunes y Tatiana Sanches. 2018. La contribución de la alfabetización informacional a la Ciencia Abierta. En Ibersid: Revista de Sistemas de Información Y Documentación. Vol. 12, no. 1, 59-67. <https://www. ibersid.eu/ojs/index.php/ibersid/article/view/4437> [Consulta: 25 octubre 2021].

»Machin-Mastromatteo, Juan Daniel y Jesús Lau. 2015. The arrival of information literacy. En Information Development. Vol. 31, no. 2, 190-193. <https://doi. org/10.1177/0266666915569147>

" Majid, Shaheen, Yun-Ke Chang, Hnin Nu Aye, Ma May Win Khine y Yu Wai San. 2015. Analyzing publishing trends in information literacy literature: A bibliometric study. En Malaysian Journal of Library \& Information Science. Vol. 20, no. 2, 51-66. <https://mjlis.um.edu.my/article/view/1766> [Consulta: 17 febrero 2021].

" Onyancha, Omwoyo Bosire. 2020. Knowledge visualization and mapping of information literacy, 1975-2018. En IFLA Journal. Vol. 46, no. 2, 107-123. <https:// doi.org/10.1177/0340035220906536>

»Ortoll, Eva. 2003. Gestión del conocimiento y competencia informacional en el puesto de trabajo. UOC. <https://www.uoc.edu/dt/20343/index.html> [Consulta: 17 febrero 2021].

"Park, Myung-Kyu y Heejung Kim. 2011. A bibliometric analysis of the literature on information literacy. En Journal of the Korean Society for information Management. Vol. 28, no. 2, 53-63. <https://doi.org/10.3743/KOSIM.2011.28.2.053> 
"Pinto, María y Alejandro Uribe-Tirado. 2017. Presencia de la formación en competencias informacionales (Alfin) en las bibliotecas nacionales iberoamericanas. En Investigación Bibliotecológica. Archivonomía, Bibliotecología e Información. Vol. 31, no. 71, <https://doi.org/10.22201/iibi.0187358xp.2017.71.57811>

"Pinto, María, María Isabel Escalona-Fernández y Antonio Pulgarín. 2013. Information literacy in social sciences and health sciences: a bibliometric study (1974-2011). En Scientometrics. Vol. 95, no.3, 1071-1094. <https://doi.org/10.1007/ S11192-012-0899-y>

»Ponjuán, Gloria, María Pinto y Alejandro Uribe-Tirado. 2015. Conceptualización y perspectivas de la alfabetización informacional en Iberoamérica: un estudio Delphi. En Information Research. Vol. 20, no. 3. <http://www.informationr.net/ ir/20-3/paper68o.html\#.Vli1Ydlvfcu> [Consulta: 17 febrero 2021].

»Rader, Hannelore. 2002. Information Literacy 1973-2002: A Selected Literature Review. En Library Trends. Vol. 51, no. 2, 242-259. <https://www.ideals.illinois.edu/ bitstream/handle/2142/8464/librarytrendsv51i2i_opt.pdf?sequence $=1 \& i \% 20$ sAllowed $=y>$ [Consulta: 17 febrero 2021].

»Sales, Dora 2020. Definición de alfabetización informacional de CILIP, 2018. En Anales de Documentación. Vol. 23, no. 1). <https://doi.org/10.6018/analesdoc.373811>

»Sample, Angela. 2020. Historical development of definitions of information literacy: A literature review of selected resources. En The Journal of Academic Librarianship. Vol. 46, no. 2, 102-116. <https://doi.org/10.1016/j.acalib.2020.102116>

»Stopar, Karmen y Tomaz Bartol. 2019. Digital competences, computer skills and information literacy in secondary education: mapping and visualization of trends and concepts. En Scientometrics. Vol. 118, no. 2, 479-498. <https://doi. org/10.1007/s11192-018-2990-5>

»Uribe-Tirado, Alejandro. 2012. Empleo de las tecnologías en los programas de alfabetización informacional. En Tarango, Javier y Gabriela Mendoza Guillén, eds. Didáctica básica para la alfabetización informacional. Buenos Aires: Alfagrama Ediciones. p. 347-382. <http://eprints.rclis.org/18183/> [Consulta: $17 \mathrm{fe}$ brero 2021].

"Uribe-Tirado, Alejandro y Joel Alhuay-Quispe. 2017. Estudio métrico de ALFIN en Iberoamérica: De la bibliometría a las altmetrics. En Revista Española de Documentación Científica. Vol. 40, no. 3, e18o. <https://doi.org/10.3989/redc.2017.3.1414>

»Uribe-Tirado, Alejandro, Luciano Gallón, Álvaro Monterroza-Ríos, Álvaro Quintero-Posada y Gabriel Vélez-Cuartas. 2020. Datos, información, conocimiento: otra cruel pedagogía del virus. En Polifonía para pensar una pandemia. Medellín: Universidad de Antioquia, Fondo Editorial FCSH de la Facultad de Ciencias Sociales y Humanas. p. 67-88 <http://eprints.rclis.org/40763/> [Consulta: 17 febrero 2021].

»Webber, Sheila y Bill Johnston. 2002. Information literacy: the social action agenda. En National Information Literacy Conference (5th: 30 noviembre-1 diciembre 2001: Adelaide). Trabajos presentados. Adelaide: University of South Australia Library. p. 68-8o. <https://eprints.whiterose.ac.uk/8385/> [Consulta: 17 febrero 2021]. 